\title{
The Writing on the Wall
}

Brian M. Wolpin, M.D., M.P.H., Peter F. Weller, M.D., Joel T. Katz, M.D., Bruce D. Levy, M.D., and Joseph Loscalzo, M.D., Ph.D.

Clinical Pathological Conference Series, Department of Medicine, Brigham and Women's Hospital (B.M.W., J.T.K., B.D.L., J.L.); Beth Israel Deaconess Medical Center (P.F.W.); and Harvard Medical School (B.M.W., P.F.W., J.T.K., B.D.L., J.L.) - all in Boston

\begin{abstract}
A 52-year-old man presented to the emergency department with abdominal discomfort. His symptoms began 5 weeks earlier, when nonradiating discomfort developed in the epigastric region. The pain worsened after eating and was associated with nausea and bloating that were partially relieved by eructation. Two weeks before presentation, the patient's pain became more localized to the periumbilical area and was accompanied by early satiety and multiple episodes of vomiting. He had no fever, diarrhea, tenesmus, melena, or hematochezia.
\end{abstract}

The patient's report of epigastric and periumbilical abdominal discomfort, accompanied by bloating, nausea, and early satiety, focuses attention on the stomach, proximal small bowel, and pancreaticobiliary tree. I am particularly concerned about a process inhibiting transit through the small bowel, such as a partial small-bowel obstruction. Although conditions such as myocardial ischemia and lower-lobe pneumonia can also be manifested as upper abdominal discomfort, the duration of the symptoms for several weeks and the absence of other associated symptoms argue against these disorders.

The medical history included mild intermittent asthma that was diagnosed 20 years earlier and required only very occasional use of inhaled beta-agonists, obstructive sleep apnea, hypertension, dyslipidemia, type 2 diabetes mellitus that was well controlled with oral agents, hypothyroidism, and gout. A diagnosis of chronic idiopathic angioedema-urticaria was established 11 years earlier, after the patient presented with sporadic episodes of cutaneous swelling and hives. Skin testing did not identify an allergic trigger, and levels of serum complement were normal. Symptoms were alleviated with cetirizine and ranitidine. Other medications included lisinopril (initiated 18 months before presentation), simvastatin, metformin, levothyroxine, and colchicine. The patient was married, with two children, and worked as a pension-fund manager. He drank five beers and smoked one pack of cigarettes weekly. He did not use illicit drugs. He had traveled to Panama 5 years before presentation and to Scotland 1 year before presentation.

Address reprint requests to Dr. Levy at the Department of Medicine, Brigham and Women's Hospital, 75 Francis St., Boston, MA 02115, or to blevy@ partners.org.

This case was originally presented at the Clinicopathological Conference of the Department of Medicine at Brigham and Women's Hospital.

No potential conflict of interest relevant to this article was reported. 
The patient's hypothyroidism and angioedema-urticaria are of particular interest, given his presentation. It would be useful to know whether his thyrotropin level has been checked recently. Hypothyroidism, if severe, could explain some of the patient's symptoms, with poor gastric emptying and slowed transit through the small bowel.

Angioedema - resulting from mast-cell-mediated or bradykinin-mediated increases in vascular permeability - is characterized by the rapid onset of self-limited, localized areas of swelling that are asymmetric and nondependent and can involve the gastrointestinal tract. An inherited form of angioedema is attributable to low levels of $\mathrm{C} 1$ inhibitor, although this form of angioedema is not accompanied by urticaria and is typically manifested at a young age. Angiotensin-converting-enzyme (ACE) inhibitors have also been associated with angioedema because they can increase levels of bradykinin, but this patient's symptoms of angioedema began many years before the initiation of treatment with an ACE inhibitor. This patient has received a diagnosis of chronic idiopathic angioedema-urticaria, which is a diagnosis of exclusion, made after other causes of angioedema have been eliminated. This condition is of direct relevance to the patient's presentation. Symptoms can typically be managed with administration of antihistamines and rarely involve the gastrointestinal tract.

Physical examination revealed a well-nourished man who was not in acute distress. His temperature was $36.1^{\circ} \mathrm{C}$, heart rate 77 beats per minute, blood pressure $106 / 60 \mathrm{~mm} \mathrm{Hg}$, respiratory rate 20 breaths per minute, and oxygen saturation $98 \%$ while he was breathing ambient air. The sclerae were anicteric. The neck was supple, without palpable lymphadenopathy or thyromegaly. The lungs were clear on auscultation, and the cardiac examination was normal. The abdomen was distended, but without bulging flanks or fluid wave. Bowel sounds were normal. Palpation of the abdomen revealed mild, diffuse tenderness without rebound, guarding, or organomegaly. No luminal irregularities were detected on rectal examination, and a guaiac test of stool was negative. The patient's arms and legs were warm and well perfused. Skin and neurologic examinations were unremarkable.

The examination is helpful in reducing concern about catastrophic complications involving the gastrointestinal tract that might arise in conditions such as acute or chronic appendicitis, acute cholecystitis, or bowel perforation. Abdominal distention can result from the presence of air, fluid, organomegaly, or a mass. Given the absence of palpable hepatosplenomegaly or masses, with no apparent evidence of ascites and no signs of peritoneal involvement, the distention in this case seems most consistent with the presence of air in the bowel, perhaps associated with an ileus or bowel obstruction. The absence of palpable splenomegaly is an important finding, since this condition can result in early satiety and discomfort in the epigastric region and the left upper quadrant. The differential diagnosis of the patient's symptoms remains quite broad. The initial evaluation would be facilitated by a complete blood count, tests for liver function and levels of pancreatic enzymes and thyrotropin, and abdominal radiographs obtained with the patient in the upright and supine positions.

The leukocyte count was 9480 cells per cubic millimeter, with $31 \%$ neutrophils, $24 \%$ lymphocytes, $6 \%$ monocytes, and $39 \%$ eosinophils. The hematocrit was $32.6 \%$, with a mean corpuscular volume of $88 \mathrm{fl}$. The platelet count was 295,000 per cubic millimeter. A 
peripheral-blood smear showed no dysplastic cells. The serum sodium level was $139 \mathrm{mmol}$ per liter; potassium, $3.8 \mathrm{mmol}$ per liter; blood urea nitrogen, $16 \mathrm{mg}$ per deciliter $(5.7 \mathrm{mmol}$ per liter); creatinine, $1.3 \mathrm{mg}$ per deciliter (115 $\mu \mathrm{mol}$ per liter); glucose, $126 \mathrm{mg}$ per deciliter (7 mmol per liter); calcium, $9.0 \mathrm{mg}$ per deciliter ( $2.2 \mathrm{mmol}$ per liter); total protein, $6.6 \mathrm{~g}$ per deciliter; and albumin, $4.1 \mathrm{~g}$ per deciliter. Liver function and lipase levels were normal. The thyrotropin level was $3.3 \mathrm{mIU}$ per liter (normal range, 0.5 to 5.0). The prothrombin and partial thromboplastin times were not prolonged. An electrocardiogram and a chest radiograph were unremarkable.

The most notable laboratory finding is the high proportion of eosinophils at $39 \%$, with a calculated absolute eosinophil count of about 3700 cells per cubic millimeter. Eosinophilia is commonly defined as an elevated percentage of eosinophils, with an absolute eosinophil count of more than 500 cells per cubic millimeter. In this patient, who has gastrointestinal discomfort and a history of asthma, has traveled internationally, and has angioedema, several disorders associated with eosinophilia warrant consideration.

Although asthma itself rarely results in peripheral-blood eosinophil counts of more than 800 cells per cubic millimeter, a possibility is Churg-Strauss syndrome, a condition characterized by asthma, eosinophilia, and sinus disease. Although the patient has a history of asthma, the asthma associated with Churg-Strauss syndrome typically develops later in life and becomes progressively more severe, often requiring treatment with oral corticosteroids; a variety of other disease manifestations typically follow, such as peripheral neuropathy, skin lesions, transient pulmonary infiltrates, and renal dysfunction. The gastrointestinal tract may be involved, and this is typically manifested as abdominal discomfort, diarrhea, and bleeding. Evidence supportive of this diagnosis includes a positive finding for perinuclear antineutrophil cytoplasmic antibodies, which are present in approximately $50 \%$ of patients, or a biopsy specimen of an affected organ that reveals eosinophil-associated vasculitis.

Given the patient's travel to Panama 5 years before presentation, strongyloidiasis should be considered; it can have a long latency period between infection and the development of symptoms, commonly causes eosinophilia, and infects the bowel. Infection with the human immuno-deficiency virus (HIV) is also associated with eosinophilia and gastrointestinal infections, but eosinophilia is more often seen in patients with advanced disease.

Further consideration of the patient's history of angioedema introduces the possibility of a rare syndrome characterized by recurrent episodes of angioedema and peripheral eosinophilia. However, this syndrome is also manifested as fever, weight gain, and marked leukocytosis (none of which are reported in this patient), as well as elevated serum levels of $\operatorname{IgM}$.

Finally, an important consideration is an organ-confined disease, such as eosinophilic gastroenteritis. This disorder is associated with peripheral-blood eosinophilia and bowel dysfunction resulting from infiltration of the bowel wall by eosinophils and can be manifested as symptoms of intestinal obstruction. 
Abdominal imaging and evaluation for parasitic disease, including stool studies for ova and parasites and a serologic test for Strongyloides stercoralis, would be reasonable next steps in the patient's evaluation.

The patient was admitted for further evaluation. Measurement of strongyloides antibodies by enzyme-linked immunosorbent assay (ELISA) revealed a count of less than 1 ELISA unit. The results of a single stool study for ova and parasites was negative, and serum antineutrophil cytoplasmic antibodies were undetectable. Computed tomography (CT) of the abdomen and pelvis revealed circumferential wall thickening of the jejunum, with proximal dilatation and complete decompression of the distal small bowel loops (Fig. 1). No discrete transition point or mass was identified. A moderate amount of ascites was present in the pelvis and around the liver and spleen.

The evaluation for parasitic infection was unrevealing, although several stool examinations for strongyloides larvae may be needed when serologic-test results are not available or the host is immunocompromised. The CT findings are nonspecific and may be seen in cases of inflammatory bowel disease, intestinal ischemia, mechanical obstruction, infection, malignant disease, and infiltrating disorders; however, marked peripheral-blood eosinophilia would not be expected with inflammatory bowel disease, ischemia, or mechanical obstruction. Strongyloides may reside in the duodenojejunal regions of the small bowel, but this parasite does not cause jejunal-wall thickening. In contrast, cancer and infiltrating disorders, particularly eosinophilic gastroenteritis, can cause a peripheral-blood eosinophilia and involve the bowel wall, leading to a thickened appearance on imaging studies. Analysis of ascitic fluid and direct visualization of the bowel with tissue biopsy are indicated for further evaluation of these entities.

Ultrasound-guided paracentesis failed to procure ascitic fluid for analysis. Esophagogastroduodenoscopy with push enteroscopy revealed a normal-appearing esophagus, stomach, pylorus, duodenum, and jejunum. Analyses of multiple random biopsy specimens obtained from the jejunum revealed normal small-intestinal mucosa.

I remain concerned about the possibility of an infiltrative disorder or a cancer that could not be visualized in a mucosal-biopsy specimen. A full-thickness biopsy specimen of the jejunum is warranted to look for a process involving the submucosa.

An exploratory laparotomy was performed, and a full-thickness biopsy specimen of the jejunum was obtained. The specimen showed infiltration of the muscularis propria and subserosa by sheets of eosinophils (Fig. 2). Flow cytometry did not reveal features consistent with involvement by a B-cell lymphoproliferative disorder. Ascitic fluid obtained at the time of surgery contained 13,200 white cells per cubic millimeter, with $68 \%$ eosinophils, and no cells that appeared to be malignant; a stain for acid-fast bacilli was negative. Eosinophilic gastroenteritis was diagnosed, and treatment with $60 \mathrm{mg}$ of prednisone orally per day was initiated, with rapid and dramatic relief of symptoms and reduction of peripheral eosinophilia. Further studies revealed ascitic-fluid cultures that were negative for bacteria, mycobacteria, and fungi; normal serum levels of vitamin $\mathrm{B}_{12}$, total $\mathrm{IgE}$, and tryptase; and a normal echocardiogram. Peripheral-blood testing by means of polymerase-chain-reaction 
assay was negative for the fusion gene FIP1L1-PDGFRa (Fip1-like 1-platelet-derived growth factor alpha), a constitutively active tyrosine kinase implicated in the pathogenesis of chronic eosinophilic leukemia. The patient was slowly weaned from the prednisone over the course of 4 months, without disease recurrence. Six months after his diagnosis, he remains symptom-free, with a normal eosinophil count, and continues to be followed closely.

\section{Commentary}

The evaluation of this patient illustrates a stepwise integration of information from the history, physical examination, laboratory tests, and imaging studies that led to a relatively narrow differential diagnosis, even though the clinical scenario was complex. The patient's history and physical examination focused generally on the stomach and small bowel and more specifically on processes that lead to obstruction of these organs. This differential diagnosis was narrowed after laboratory testing revealed marked peripheral-blood eosinophilia. Another important piece of the puzzle was supplied by the imaging studies, which showed thickening of the wall of the small bowel. Among the diseases leading to symptoms of gastrointestinal obstruction and eosinophilia, a limited number of disorders primarily eosinophilic gastroenteritis and malignant diseases - are also likely to cause thickening of the small bowel in the absence of other systemic disease. Ultimately, it was necessary to examine a full-thickness biopsy specimen to diagnose eosinophilic gastroenteritis involving the submucosal layers of the jejunum.

Eosinophilic gastroenteritis is characterized by an eosinophil-rich inflammation in the wall of the stomach or small bowel. ${ }^{1}$ Before this diagnosis is established, it is necessary to rule out other causes of eosinophilia, such as drug reaction, parasitic infection, and cancer. In addition, the involvement of other organs must be ruled out, since multiorgan involvement indicates the presence of an alternative hypereosinophilic syndrome, such as eosinophilic leukemia, hypereosinophilic syndrome, or the Churg-Strauss syndrome. ${ }^{2}$ If the diagnosis of eosinophilic gastroenteritis is suspected, further investigation to rule out other hypereosinophilic syndromes should routinely include a peripheral-blood smear, serum levels of tryptase and vitamin $B_{12}$ (both of which can be elevated in the myeloproliferative variant of hypereosinophilic syndrome), and a chest radiograph. ${ }^{1}$ To detect myocardial damage, a serum troponin assay and an echocardiographic study should be performed. If there is reason to suspect a myeloproliferative variant of hypereosinophilic syndrome or another hematologic cancer (on the basis of lymphadenopathy, splenomegaly, morphologic abnormalities of peripheral-blood cells, or elevated serum levels of vitamin $\mathrm{B}_{12}$ or tryptase), bone marrow analysis should be considered. ${ }^{3}$ Biopsy of other potentially involved tissues should also be performed, as guided by clinical findings.

For patients with eosinophilic gastroenteritis, presenting symptoms typically vary in accordance with the layer of bowel wall involved. ${ }^{4}$ Mucosa-predominant disease is manifested as nonspecific abdominal discomfort, malabsorption, and protein-losing enteropathy, whereas muscularis-predominant disease leads to symptoms of obstruction, such as early satiety, bloating, and nausea. In contrast, subserosa-predominant disease more commonly results in eosinophilic ascites and markedly elevated counts of eosinophils in peripheral blood. This patient had involvement of both the muscularis and subserosal layers 
of the small bowel, with the characteristic obstructive symptoms, eosinophilic ascites, and elevated eosinophil count in peripheral blood. Mucosal biopsies are frequently unrevealing in patients with eosinophilic gastroenteritis involving the deeper layers of the bowel wall; more invasive procedures are needed to confirm the diagnosis. ${ }^{5}$

The cause of eosinophilic gastroenteritis is not well understood. Although many patients have a history of allergic disease, such as asthma, allergic rhinitis, or atopy, the extent of the role played by food allergies in the pathogenesis of eosinophilic gastroenteritis remains controversial. ${ }^{6}$ Skin-prick testing can be performed with a panel of allergens to facilitate directed trials of food avoidance, but the success rate of such dietary modification is not well characterized. ${ }^{7}$ Patients with an elevated serum level of total IgE appear more likely to have an underlying allergic trigger for their eosinophilic gastroenteritis and may therefore have a better response to dietary modification. ${ }^{1}$ Elevated levels of pro-eosinophilic cytokines, including interleukin-5, are thought to mediate the eosinophilic infiltration that results in response to allergic stimuli and suggest a possible future mechanism for therapeutic intervention. $^{8-10}$

In rare cases, patients with eosinophilic gastroenteritis have a clonal disorder that is better classified as chronic eosinophilic leukemia with gastrointestinal involvement. ${ }^{3}$ In a subgroup of these patients, the malignant clone has an interstitial deletion on chromosome 4q12, leading to fusion of the FIPIL1 and PDGFRa genes. ${ }^{11}$ The FIP1L1-PDGFRa fusion protein produced by this interstitial deletion is a constitutively activated tyrosine kinase that responds to treatment with imatinib mesylate. ${ }^{11,12}$ Commercial testing for this interstitial deletion is available and should be performed in patients with suspected eosinophilic gastroenteritis, as was done in this patient.

Treatment for patients with eosinophilic gastroenteritis typically entails a trial of dietary avoidance of food allergens identified clinically or with the use of skin-prick testing. If symptoms persist or are severe, treatment with corticosteroids is recommended. ${ }^{7}$ When corticosteroids are required, a dose of 20 to $40 \mathrm{mg}$ of prednisone daily is the typical starting dose, which is then tapered over the course of several months. Although few studies describe the natural history of eosinophilic gastroenteritis, approximately half of patients are thought to have a waxing and waning course, requiring periodic treatment. ${ }^{4,7}$ Data are limited on how best to manage the care of patients in whom corticosteroid therapy fails or in whom side effects occur after long-term use, but cromolyn, leukotriene inhibitors, and immunosuppressants have been used with some success in small numbers of patients. ${ }^{7,13,14}$ Although eosinophilic gastroenteritis is defined by the exclusive involvement of the gastrointestinal tract and no other organs, lifelong follow-up by clinicians familiar with this disease is important, since in rare cases it is the initial manifestation of a progressive, multiorgan hypereosinophilic syndrome. The team caring for this patient successfully integrated the disparate features of the patient's presentation and confirmed a diagnosis of submucosal eosinophilic gastroenteritis by reading the writing on the wall.

\section{References}

1. Rothenberg ME. Eosinophilic gastrointestinal disorders (EGID). J Allergy Clin Immunol. 2004; 113:11-28. [PubMed: 14713902] 
2. Klion AD, Bochner BS, Gleich GJ, et al. Approaches to the treatment of hypereosinophilic syndromes: a workshop summary report. J Allergy Clin Immunol. 2006; 117:1292-302. [PubMed: 16750989]

3. Martinelli G, Rondoni M, Ottaviani E, Paolini S, Baccarani M. Hypereosinophilic syndrome and molecularly targeted therapy. Semin Hematol. 2007; 44(Suppl 2):S4-S16. [PubMed: 17292736]

4. Méndez-Sanchez N, Chávez-Tapia NC, Vazquez-Elizondo G, Uribe M. Eosinophilic gastroenteritis: a review. Dig Dis Sci. 2007; 52:2904-11. [PubMed: 17429740]

5. Yun MY, Cho YU, Park IS, et al. Eosinophilic gastroenteritis presenting as small bowel obstruction: a case report and review of the literature. World J Gastroenterol. 2007; 13:1758-60. [PubMed: 17461485]

6. Justinich C, Katz A, Gurbindo C, et al. Elemental diet improves steroid-dependent eosinophilic gastroenteritis and reverses growth failure. J Pediatr Gastroenterol Nutr. 1996; 23:81-5. [PubMed: 8811528]

7. Caldwell JH. Eosinophilic gastroenteritis. Curr Treat Options Gastroenterol. 2002; 5:9-16. [PubMed: 11792233]

8. Jaffe JS, James SP, Mullins GE, Braun-Elwert L, Lubensky I, Metcalfe DD. Evidence for an abnormal profile of interleukin-4 (IL-4), IL-5, and gamma-interferon (gamma-IFN) in peripheral blood T cells from patients with allergic eosinophilic gastroenteritis. J Clin Immunol. 1994; 14:299_ 309. [PubMed: 7814459]

9. Sutton SA, Assa' ad AH, Rothenberg ME. Anti-IL-5 and hyper eosinophilic syndromes. Clin Immunol. 2005; 115:51-60. [PubMed: 15870021]

10. Rothenberg ME. Eosinophilia. N Engl J Med. 1998; 338:1592-600. [PubMed: 9603798]

11. Cools J, DeAngelo DJ, Gotlib J, et al. A tyrosine kinase created by fusion of the PDGFRA and FIP1L1 genes as a therapeutic target of imatinib in idiopathic hypereosinophilic syndrome. N Engl J Med. 2003; 348:1201-14. [PubMed: 12660384]

12. Pitini V, Sturniolo G, Cavallari V, Arrigo C. Rapid reversion of eosinophilic gastroenteritis associated with FIP1L1-PDGFRA fusion after targeted therapy with imatinib. Br J Haematol. 2006; 132:123. [PubMed: 16398645]

13. Urek MC, Kujundzić M, Banić M, Urek R, Veic TS, Kardum D. Leukotriene receptor antagonists as potential steroid sparing agents in a patient with serosal eosinophilic gastroenteritis. Gut. 2006; 55:1363-4. [PubMed: 16905705]

14. Bolukbas FF, Bolukbas C, Uzunkoy A, Baba F, Horoz M, Ozturk E. A dramatic response to ketotifen in a case of eosinophilic gastroenteritis mimicking abdominal emergency. Dig Dis Sci. 2004; 49:1782-5. [PubMed: 15628703] 

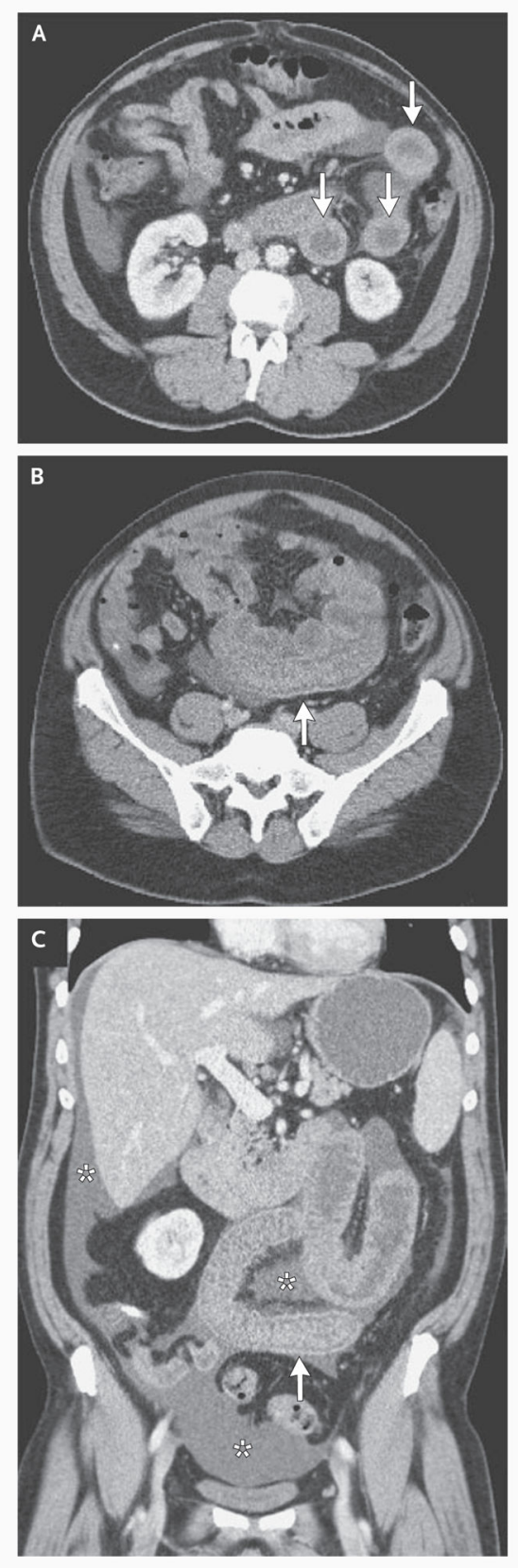

Figure 1. CT Images of the Abdomen and Pelvis

Axial images (Panels A and B) and a coronal image (Panel C) show circumferential wall thickening in a long segment of jejunum (Panel A, arrows). Mural stratification with relatively increased enhancement of the mucosa and serosa suggests bowel-wall edema (Panels B and C, arrows). A moderate volume of ascites is seen in the small-bowel mesentery, perihepatic space, and pelvis (Panel C, asterisks). 

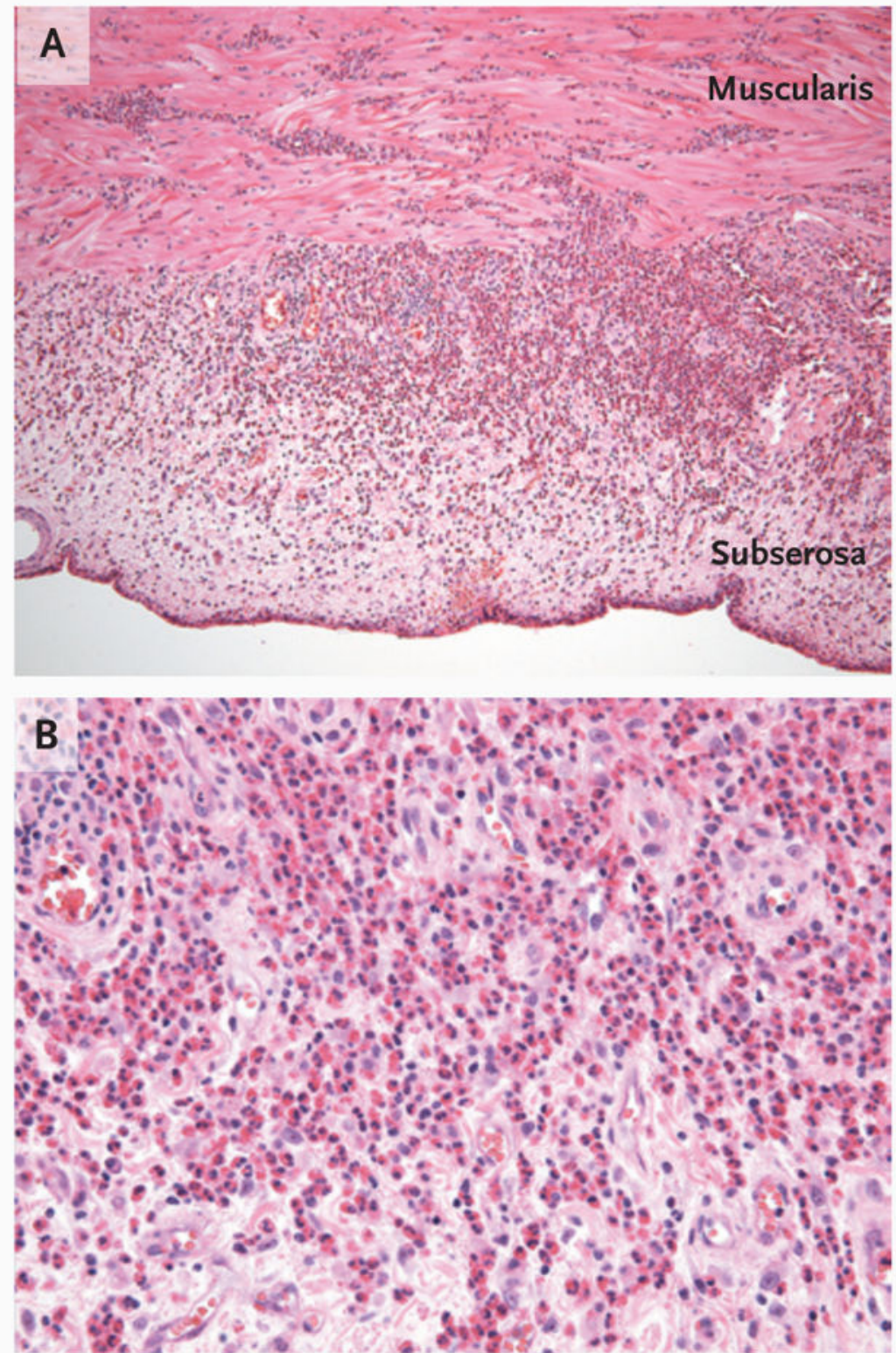

Figure 2. Full-Thickness Biopsy Specimen of the Jejunum

A full-thickness biopsy specimen of the jejunum (Panel A, hematoxylin and eosin) shows infiltration of the muscularis propria and subserosa by sheets of eosinophils, with a markedly thickened and edematous subserosa. A high-power view of the subserosa (Panel B) shows numerous eosinophils, which are characteristically deep pink on staining with hematoxylin and eosin. 